\title{
Enabling Tunable Hydrophilicity of PDMS via Metal-ligand Co- ordinated Dynamic Networks
}

\author{
Xinyue Zhang Ralph Crisci John A. Finlay Hongyi Cai Anthony S. Clare Zhan Chen Meredith N.
} Silberstein*

Xinyue Zhang, Hongyi Cai

Department of Materials Science and Engineering, Cornell University, Ithaca, NY 14850, USA

Ralph Crisci, Prof. Zhan Chen

Department of Chemistry, University of Michigan, Ann Arbor, MI 48109, USA

Dr. John A. Finlay, Prof. Anthony S. Clare

School of Natural and Environmental Sciences, Newcastle University, Newcastle Upon Tyne, NE1 7RU, UK

Prof. Meredith N. Silberstein

Sibley School of Mechanical and Aerospace Engineering, Cornell University, Ithaca, NY 14853, USA

Email Address: meredith.silberstein@cornell.edu

Keywords: dynamic network; metal-ligand coordination; polymer hydrophilicity; PDMS

Polydimethylsiloxane (PDMS) has been widely used in various fields due to its appealing physical and chemical properties. However, its high hydrophobicity not only yields poor adhesion to substrates but also facilitates undesired adsorption of substances such as proteins, biofoulers, etc., which limits the performance and lifetime of PDMS. Moreover, traditional surface modification techniques are often not efficient on PDMS surfaces because of the surface reconstruction. Although new methods involving chemical modification have been developed, most of them require complicated procedures and equipment. To overcome this challenge, we incorporate metal-ligand coordination, a non-covalent interaction bearing polar functionality, into PDMS, which exposes the hydrophilicity progressively upon dynamic bond breakage and reformation. We demonstrate that the hydrophilicity of coordinated PDMS can be tailored by the choice of network structure, counter anions, and metal cations, which yield distinct network dynamics. The wetting mechanism is discussed in the context of chain reconfiguration and surface reconstruction. We also show that a properly designed metal-ligand coordinated PDMS has potential as a superior marine fouling release coating by weakening diatom attachment. Through this paper, we introduce a new concept for tuning material hydrophilicity via dynamic polar functionalities, which is applicable to a wide range of polymers.

\section{Introduction}

Poly(dimethylsiloxane) (PDMS) is one of the most widely used polymers with great performance for both commercial and cutting-edge research technologies. It is low cost, flexible, chemically inert, biocompatible, and easy to fabricate.[1, 2, 3] Benefiting from its attractive physical and chemical properties, plus low manufacturing cost, PDMS serves as an essential substrate or coating material in various fields.[4, 5, 6] For example: PDMS outperforms glass and other polymers as a critical substrate material in fabricating microfluidic devices for biomedical applications; $[7,8]$ it is used as a substrate in flexible electronics for developing wearable devices and soft robotics; $[9,10]$ PDMS is coated on fiber optic sensors to improve the performance and durability; $[11,12]$ furthermore, PDMS is a fouling-release coating for marine antifouling. [13, 14]

A primary shortcoming of PDMS, which often hinders its performance and shortens the lifetime of the product, is the surface properties. This is mainly due to two reasons: first, the low surface energy of PDMS $(\sim 20 \mathrm{mN} / \mathrm{m})$ yields weak interactions with other materials at the interface; second, the presence of methyl side groups results in inherently high hydrophobicity (contact angle $\sim 110 \pm 10^{\circ}$ ), making it challenging to wet by aqueous solutions and favoring undesired adsorption of proteins and biofoulers. [15, 16, 17] Therefore, treatments are often necessary to improve the surface properties of PDMS before applying it as a substrate or coating. The most well-established surface treatment technique is oxidizing the surface by exposing it to plasma or ultraviolet (UV) radiation, which replaces the methyl groups with hydroxyl groups, generating a more hydrophilic surface.[18, 19] However, hydrophobic recovery happens quickly due to PDMS surface reconstruction, with short mobile PDMS chains from the material bulk 
covering up the thermodynamically unstable surface and restoring the hydrophobic surface.[20] Even worse, a brittle silica layer is created on the surface after such treatment, which is prone to cracking.[18] In order to produce a long-lasting hydrophilic surface, other surface modification methods such as coating metal and metal oxides via atomic layer deposition (ALD), depositing hydrophilic polymer layers via chemical vapor deposition (CVD), and grafting hydrophilic polymer brushes via plasma or UV polymerization have been investigated.[21, 22, 23, 24] Alternatively, the hydrophilicity of PDMS has been improved by altering the bulk chemistry. Hydrophilic segments have been combined with PDMS chains, for example, copolymers of poly(ethylene oxide) (PEO) and PDMS, amphiphilic polymers consisting of poly(sulfobetaine methacrylate) (PSBMA) and PDMS, and peptoid side chain functionalized PDMS have been synthesized.[2, 25, 26, 27] However, these methods usually involve complicated and expensive organic synthesis.

The hydrophilicity of a material is frequently measured using the contact angle of water droplets on the material surface by an optical tensiometer.[20, 28] According to the Owens-Wendt, Rabel, and Kaelble (OWRK) method, when applying a liquid on a solid, the solid-liquid interface energy $\gamma_{S L}$ is

$$
\gamma_{S L}=\gamma_{S}^{D}+\gamma_{S}^{P}+\gamma_{L}^{D}+\gamma_{L}^{P}-2 \sqrt{\gamma_{S}^{D} \gamma_{L}^{D}}-2 \sqrt{\gamma_{S}^{P} \gamma_{L}^{P}}
$$

where $\gamma_{S}^{D}$ and $\gamma_{S}^{P}$ refer to the dispersive and polar contributions respectively to the surface energy of a solid, and $\gamma_{L}^{D}$ and $\gamma_{L}^{P}$ refer to the dispersive and polar contributions respectively to the surface energy of a liquid.[29, 30] Combining Equation 1 with Young's equation, we obtain

$$
\cos \theta=\frac{2 \sqrt{\gamma_{S}^{D} \gamma_{L}^{D}}+2 \sqrt{\gamma_{S}^{P} \gamma_{L}^{P}}}{\gamma_{L}^{D}+\gamma_{L}^{P}}-1
$$

where $\theta$ is the contact angle at the solid-liquid interface. Equation 2 evaluates the water contact angle resulting from incorporating polar functionalities into a polymer matrix: $\theta$ decreases as $\gamma_{S}^{P}$ increases, indicating that surface is more hydrophilic. One means to increase hydrophilicity is therefore adding more polar functionality to the surface.

In this paper, instead of applying post-synthesis surface treatment or altering the backbone chemistry, we present a simple and efficient way to embed polar segments inside the PDMS matrix. Benefiting from the versatility of metal-ligand coordination, which not only provides the polar functionalities, but also imparts a dynamic nature into the coordinated network, the exposure of hydrophilicity is dominated by the network dynamics through chain motion.[31] Moreover, metal-ligand coordination enables great design flexibility by selection of ligand species, counter anions, and metal cations, which yields various network architectures, coordination strength and kinetics, and further facilitates programming the hydrophilicity of the materials for various purposes.[32, 31] We first demonstrate the feasibility of the design by comparing the ligand functionalized PDMS with different chain mobility but the same metal coordination density, and then tune the coordination strength of the model system by switching counter anions and metal cations. The network dynamics were characterized by mechanical tests, and the hydrophilicity of the coordinated PDMS was monitored by water contact angle. Sum Frequency Generation (SFG) vibrational spectroscopy, a second-order nonlinear optical spectroscopy technique, [33, 34, $35,36,37$ ] was applied to probe surface chemical structures of PDMS materials in air and in water as well as time-dependent interfacial structural changes. The water wetting mechanism is discussed in the context of network dynamics. A diatom fouling test shows the superior fouling-release properties of the cobalt coordinated PDMS coatings. The insights included in this paper introduce fundamental design ideas for improving material hydrophilicity, which is applicable to a wide range of polymers to achieve better performance. 


\section{Results and Discussion}

\subsection{Metal-Ligand Coordination in PDMS to Control Surface Hydrophilicity}

Metal-ligand coordination offers great flexibility in terms of designing the physical and chemical characteristics of a polymer network: factors such as the ligand species, the selection of counter ions, and metal cations can greatly alter the material behavior. To prove the idea that the hydrophilicity of a metalligand coordinated PDMS can be tuned by controlling the dynamics of the network, we carefully selected the structure of the model system: the PDMS backbone is free from entanglement to ensure chain mobility, and both chain ends are capped by pyridyl imine bidentate ligands to be able to coordinate with various metal salts. [38, 39, 40] Experimentally, the pyridyl imine functionalized PDMS (named PI) was synthesized through a condensation reaction between the aminopropyl terminated PDMS and 2-pyridinecarboxaldehyde, yielding a yellow colored oil-like liquid (Figure 1a).[38, 41] The structure of PI was verified by ${ }^{1} \mathrm{H}-\mathrm{NMR}$ (Figure S1). Gel permeation chromatography (GPC) shows that the number average molecular weight $\left(M_{n}\right)$ of PI is $\sim 2000 \mathrm{~g} / \mathrm{mol}$ (Figure S3), which is far below the critical entanglement value of PDMS $\left(M_{e} \sim 10^{4} \mathrm{~g} / \mathrm{mol}\right)$. [42] A divalent transition metal salt is added into the PDMS to form a metal-ligand coordinated PDMS network. One metal center is coordinated by three PI chains in an octahedral geometry, forming an end-crosslinked network. [41, 43] To create a clear contrast in PDMS chain dynamics while retaining the metal-ligand coordination density, a control system consisting of diiminopyridine tridentate ligand functionalized PDMS (named DIP) was synthesized through a condensation reaction between aminopropyl terminated PDMS and 2,6-pyridinedicarboxaldehyde, yielding a deep orange colored highly viscous liquid (Figure 1b).[44] The amine-aldehyde condensation reaction happens at both aldehyde moieties on 2,6-pyridinedicarboxaldehyde. Therefore, the diiminopyridine ligands generated not only provide the coordination ability from the electron-rich nitrogen atoms, but also connect the PDMS precursors into longer chains. The structure of DIP was verified by ${ }^{1} \mathrm{H}-\mathrm{NMR}$ (Figure S2). The higher $M_{n}$ of DIP $(\sim 9600 \mathrm{~g} / \mathrm{mol})$ compared to PI resulting from the connection of diiminopyridine ligands was confirmed by GPC (Figure S3). When divalent transition metal salt is added, one metal center is coordinated by two diiminopyridine ligands, forming an inter-crosslinked network. [45] The DIP network is expected to be less dynamic than the PI network even when the metal coordination bond dynamics are matched because the DIP chains remain constrained on two ends when a metal coordination bond opens, whereas the PI chains become dangling ends when a metal coordination bond opens. We chose $\mathrm{Zn}$ (II) as the metal cation, because it forms stable octahedral complexes with both pyridyl imine ligands and diiminopyridine ligands. $[43,44]$ Zinc tetrafluoroborate salt $\left(\mathrm{Zn}_{(}\left(\mathrm{BF}_{4}\right)_{2}\right)$ was added into the model system PI and the control system DIP to form fully crosslinked metal-coordinated PDMS networks (named PI-Zn( $\left(\mathrm{BF}_{4}\right)_{2}$ and DIP-Zn( $\left(\mathrm{BF}_{4}\right)_{2}$, respectively). In both systems, the liquid linear PDMS turns into a solid upon addition of the salt. The coordinated structures were confirmed by Fourier transform infrared (FTIR) spectroscopy (Figure 2a). All the spectra were normalized by the strong absorption band at the wavelength of $\sim 1260 \mathrm{~cm}^{-1}$, corresponding to the $\mathrm{Si}-\mathrm{CH}_{3}$ bending vibration on the PDMS backbone.[46] Imine groups have a $\mathrm{C}=\mathrm{N}$ stretching peak located at $\sim 1650 \mathrm{~cm}^{-1}$ as free ligands, and the peak shifts to lower wavelength due to the decrease of $\mathrm{C}=\mathrm{N}$ bond order when forming complexes.[47, 48] Therefore, the height of the $\mathrm{C}=\mathrm{N}$ stretching peaks at $\sim 1650 \mathrm{~cm}^{-1}$ decrease after coordination. A new peak arises at $\sim 1590 \mathrm{~cm}^{-1}$ for PI-Zn(BF $)_{2}$, and similarly, a new peak appears at $\sim 1600 \mathrm{~cm}^{-1}$ for DIP-Zn $\left(\mathrm{BF}_{4}\right)_{2}$.

To investigate the difference in the dynamic characteristics of the two $\mathrm{Zn}\left(\mathrm{BF}_{4}\right)_{2}$-coordinated networks, we performed monotonic and cyclic uniaxial tensile tests (Figure 2b). As shown in the pictures, PI-Zn(BF $)_{2}$ is soft and highly extensible, whereas DIP-Zn( $\left(\mathrm{BF}_{4}\right)_{2}$ is stiff and brittle. From the monotonic stress-strain curves, we can see that the network of PI-Zn(BF $)_{2}$ is much weaker than DIP-Zn(BF $)_{2}$, and the difference in Young's modulus (Table S1) is more than 170 times. Furthermore, when increasing the strain, $\mathrm{PI}-\mathrm{Zn}\left(\mathrm{BF}_{4}\right)_{2}$ shows pronounced softening after yield, implying chains slide past each other easily, resulting in a continuous flow of the material to release the stress.[49] On the contrary, DIP-Zn(BF 4$)_{2}$ breaks at quite a low strain, $\epsilon \approx 0.2$, without softening, implying the chains are less mobile. The more dynamic behavior of PI-Zn(BF $)_{2}$ compared to DIP- $\mathrm{Zn}\left(\mathrm{BF}_{4}\right)_{2}$ can also be seen from the stress response under 
(a)
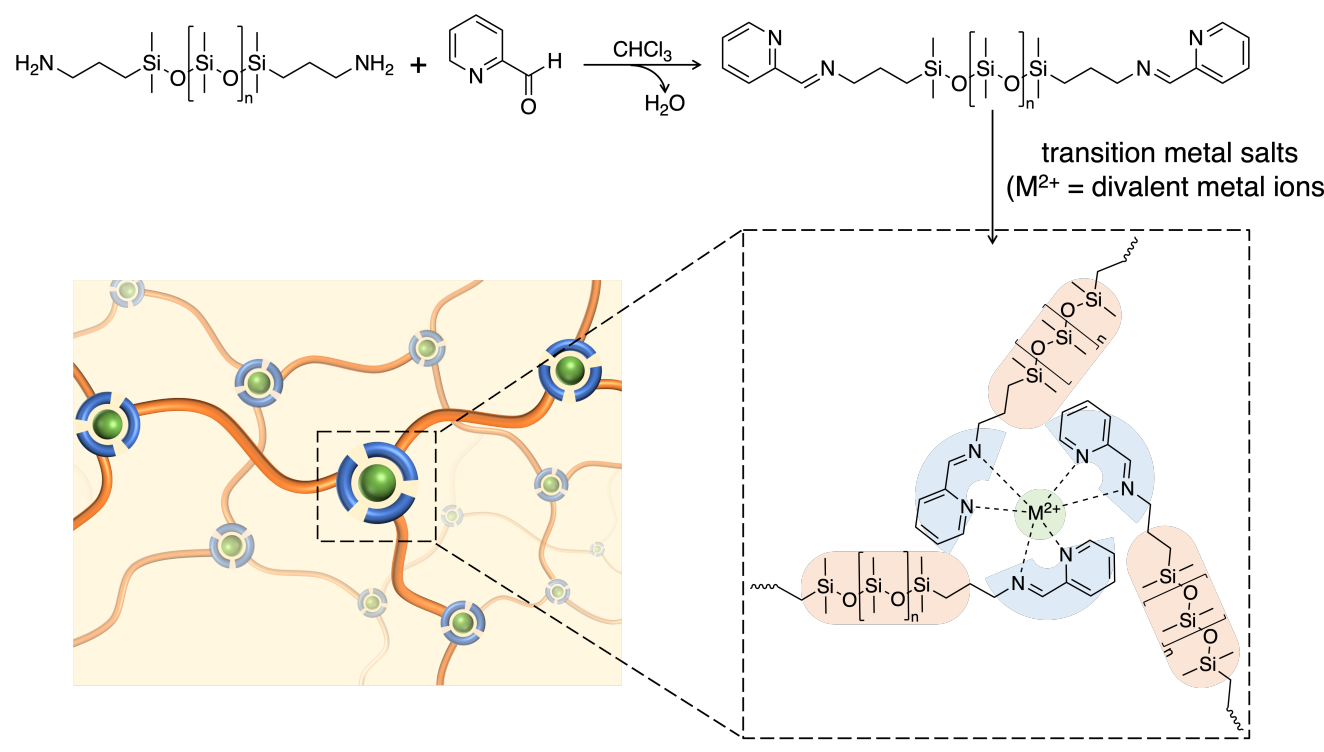

(b)
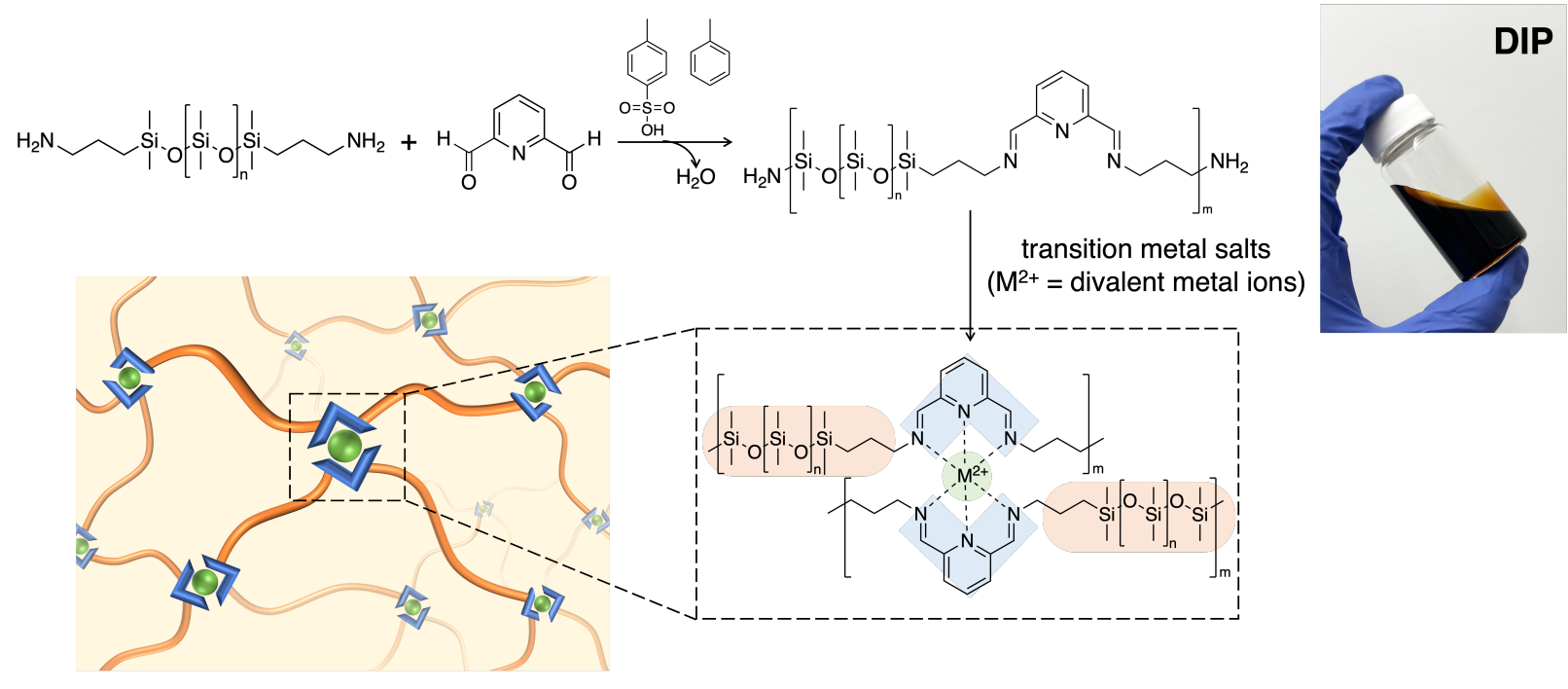

Figure 1: Synthesis of ligand functionalized PDMS and the corresponding metal complexes. (a) The model system: a condensation reaction between aminopropyl terminated PDMS and 2-pyridinecarboxaldehyde was performed to synthesize PI, generating a yellow oil-like liquid. An end-crosslinked network is formed by the coordination of PI with divalent transition metal cations. (b) The control system: a condensation reaction between aminopropyl terminated PDMS and 2,6-pyridinedicarboxaldehyde was performed to synthesize DIP, generating a deep orange colored highly viscous liquid. An inter-crosslinked network is formed by the coordination of the DIP and divalent transition metal cations. 
(a)

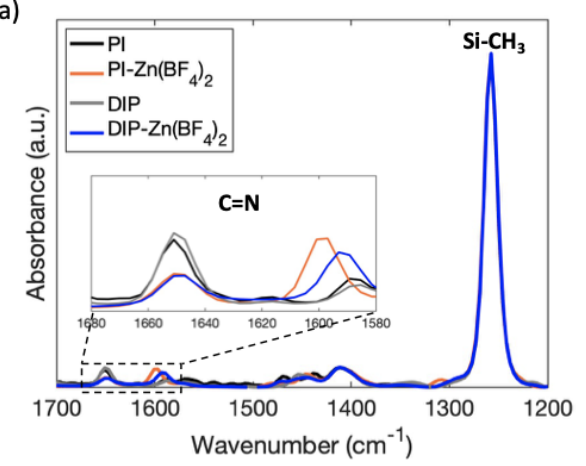

(b)

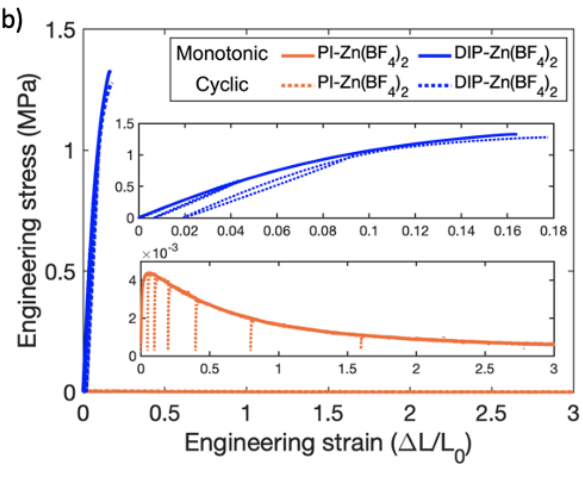

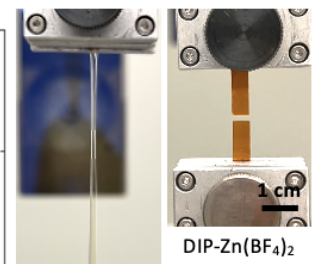

(c)

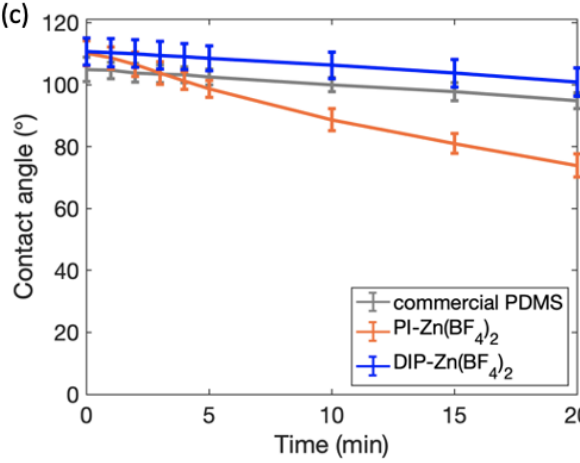

(e)

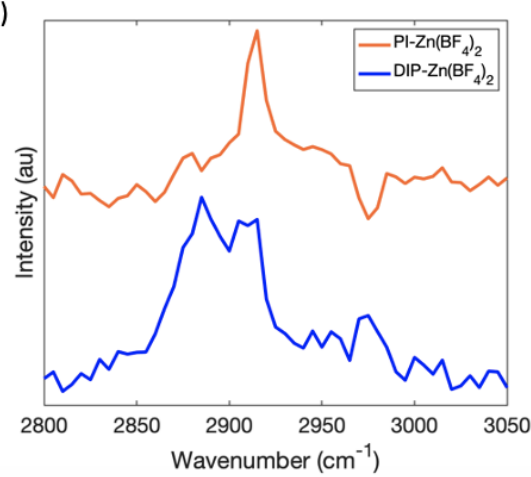

commercial PDMS

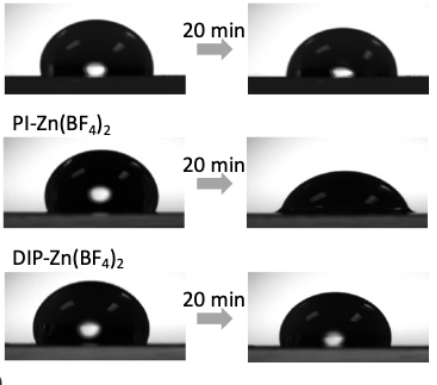

(f)

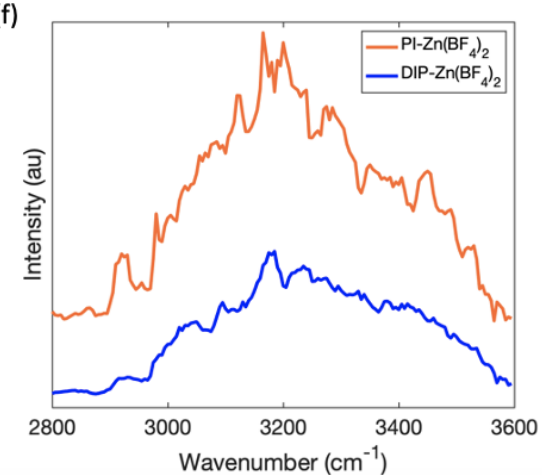

(d)

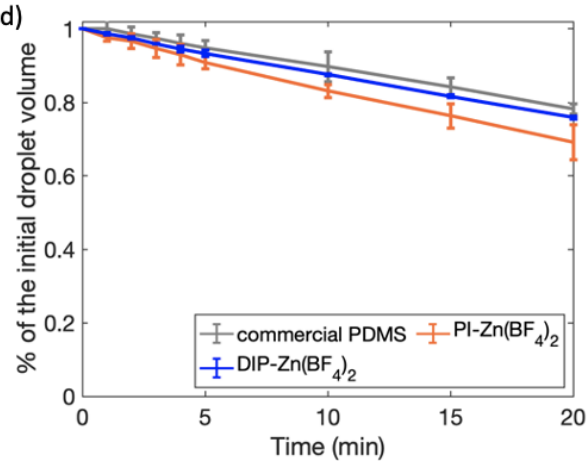

(g)

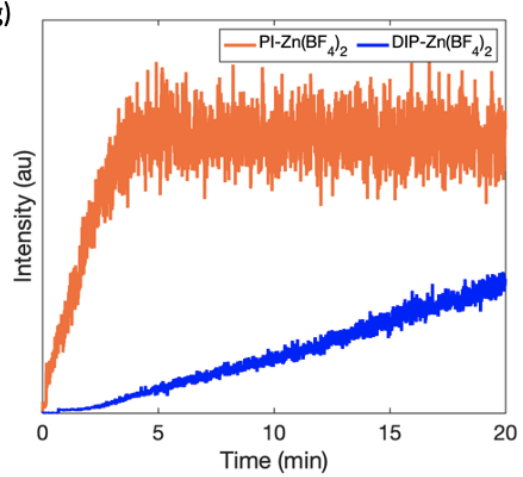

Figure 2: Comparison of $\mathrm{Zn}\left(\mathrm{BF}_{4}\right)_{2}$ coordinated PDMS networks. (a) FTIR spectra of PI and DIP before and after $\mathrm{Zn}\left(\mathrm{BF}_{4}\right)_{2}$ coordination. (b) Monotonic and cyclic tensile tests plotted as engineering stress as a function of engineering strain and photos of the specimen. Insets are the zoom-in of each material for a better view. (c) Water contact angle evolution on PDMS surfaces and photos of the interface upon droplet application and after 20 min. (d) Change of the droplet volume. (e) SFG spectra in air. (f) SFG spectra in water after $20 \mathrm{~min}$. (g) Time-dependent SFG signal intensity at $3200 \mathrm{~cm}^{-1}$.

cyclic loading. PI-Zn( $\left(\mathrm{BF}_{4}\right)_{2}$ does not show strain recovery in the unloading process, because the chains flow rapidly to accommodate the deformation, leading to plastic deformation and energy dissipation. The less dynamic DIP-Zn( $\left(\mathrm{BF}_{4}\right)_{2}$ unloads primarily elastically, showing significant strain recovery. We expect that the difference in the mechanical properties of the two materials is primarily due to network architecture, but there may also be a contribution of coordination stability resulting from the different ligand species.[50]

We characterized the hydrophilicity of $\mathrm{Zn}\left(\mathrm{BF}_{4}\right)_{2}$ coordinated PDMS networks by water contact angle tests using the sessile drop method.[51] During each measurement, a small droplet of water was deposited on the PDMS coated glass open to the ambient lab environment, and the contact angle variation was monitored by a tensiometer for 20 min (Figure 2c, Table S2). A commercial PDMS (Oomoo ${ }^{\circledR} 30$ ) was also measured as a second control. The contact angle of the commercial PDMS is $\sim 105^{\circ}$ upon water attachment, and the value drops $\sim 10^{\circ}$ over $20 \mathrm{~min}$. As seen in the photos, the contact line between the water droplet and the PDMS surface remains pinned, while the shape of the droplet is flattened. Given that no observable amount of water penetrates into the PDMS substrate, we attribute the 
decrease of contact angle to water evaporation.[52] For the dynamic system, the contact angle of PI$\mathrm{Zn}\left(\mathrm{BF}_{4}\right)_{2}$ of $\sim 110^{\circ}$ upon water attachment is within the reported range of a typical PDMS surface and indicates the surface is initially hydrophobic. [17] Interestingly, instead of having a pinned contact line, the water droplet slowly spreads out on the PI-Zn(BF $\left.)_{2}\right)_{2}$ surface. A dramatic contact angle decrease together with the expanded contact area is observed in contrast to the commercial PDMS, implying a more hydrophilic surface is rendered. Moreover, the control system DIP-Zn( $\left.\mathrm{BF}_{4}\right)_{2}$ behaves quite similarly to the commercial PDMS as expected. The contact line is pinned and the contact angle drops $\sim$ $10^{\circ}$ over $20 \mathrm{~min}$. This indicates that the presence of metal salt is not sufficient to explain the contact angle change. To better understand the interaction between water droplets and the PI-Zn(BF $)_{2}$ surface, we analyzed the change of droplet volume during the process (Figure 2d, Table S3). A steady decrease is seen for all three materials, with droplets on the PI- $\mathrm{Zn}\left(\mathrm{BF}_{4}\right)_{2}$ surface showing faster volume decrease than the two control systems. Referring to the commercial PDMS, for which the droplet volume change simply results from water evaporation, we infer that water adsorption happens on the PI$\mathrm{Zn}\left(\mathrm{BF}_{4}\right)_{2}$ surface, leading to the extra droplet volume decrease. The dynamic nature of the PI- $\mathrm{Zn}(\mathrm{BF})_{4}$ network exposes the polar metal-ligand functionality efficiently through rapid chain motion, and thus exhibits higher hydrophilicity. In contrast, the DIP- $\mathrm{Zn}\left(\mathrm{BF}_{4}\right)_{2}$ control demonstrates that introducing metalligand coordination does not necessarily alter the surface hydrophilicity. A properly designed dynamic network that facilities exposing the hydrophilic functionality is crucial.

SFG was used to study the interfacial molecular behavior of PI-Zn(BF $)_{2}$ and DIP-Zn(BF $)_{2}$ to understand the effects of ligands and their associated network architecture on such behavior. SFG spectra collected from the PI-Zn(BF $)_{2}$ and DIP-Zn $\left(\mathrm{BF}_{4}\right)_{2}$ surfaces in air (Figure 2e) are different, showing different surface structures. The PI-Zn( $\left(\mathrm{BF}_{4}\right)_{2}$ surface in air is mainly covered by $\mathrm{Si}_{-} \mathrm{CH}_{3}$ groups, as evidenced by the dominating peaks centered at $\sim 2910 \mathrm{~cm}^{-1}$ and $\sim 2960 \mathrm{~cm}^{-1}$ (which is negative due to the interference with the non-resonant background) that are contributed by the symmetric and asymmetric $\mathrm{C}-\mathrm{H}$ stretches of the $\mathrm{Si}-\mathrm{CH}_{3}$ group respectively.[53] Air is a very hydrophobic medium, thus the most hydrophobic $\mathrm{Si}-\mathrm{CH}_{3}$ group in the dynamic PI-Zn( $\left(\mathrm{BF}_{4}\right)_{2}$ segregates to cover almost the entire surface with an ordered structure. In contrast, the SFG spectrum collected from the DIP-Zn $\left(\mathrm{BF}_{4}\right)_{2}$ surface in air contains signals from other functional groups (e.g., peak $\sim 2885 \mathrm{~cm}^{-1}$ ), showing the coverage of other functional groups on the surface in addition to the $\mathrm{Si}-\mathrm{CH}_{3}$ groups, because the less dynamic DIP$\mathrm{Zn}\left(\mathrm{BF}_{4}\right)_{2}$ limits the chain arrangement. After exposure to water for $20 \mathrm{~min}$, the SFG spectrum change significantly (Figure 2f and Figure S4). The $3200 \mathrm{~cm}^{-1} \mathrm{O}-\mathrm{H}$ stretching SFG signal is contributed by the strongly hydrogen bonded water molecules at the PDMS/water interface.[54, 55] The PI-Zn(BF $)_{2}$ spectrum is strong, showing ordered interfacial water molecules at the interface, due to the now hydrophilic surface of PI-Zn(BF $)_{2}$. The SFG water spectral intensity on the DIP-Zn(BF $)_{2}$ surface is weak, consistent with it remaining hydrophobic. The SFG signal at $3200 \mathrm{~cm}^{-1}$ was monitored as a function of time to follow the surface restructuring process after the surfaces were placed in contact with water (Figure $2 \mathrm{~g}$ and Figure S5). The surface of the more dynamic PI-Zn(BF $)_{2}$ restructures more rapidly than the surface of the less dynamic DIP-Zn( $\left(\mathrm{BF}_{4}\right)_{2}$. The SFG time-dependent results are well correlated with the time-dependent contact angle evolution.

\subsection{Influence of Counter anions}

Informed by the previous results, we next tuned the hydrophilicity of the Zn(II)-pyridyl imine coordinated PDMS by varying the counter anion. Previous studies show that counter anions prompt different strength and kinetics of metal-ligand coordination by interacting with the metal cation, which then further affects the network dynamics.[56, 43] Generally, it is expected that counter anions with larger size and higher charge delocalization will interfere the metal-ligand coordination less. [57] $\mathrm{ZnCl}_{2}$ and $\mathrm{Zn}\left(\mathrm{ClO}_{4}\right)_{2}$ were incorporated into the PI to form $\mathrm{Zn}(\mathrm{II})$-coordinated PDMS (named PI-ZnCl 2 and PI-Zn(ClO $)_{2}$, respectively). The formation of these complexes was confirmed by the FTIR (Figure S6). Monotonic and cyclic uniaxial tensile tests were performed on $\mathrm{PI}-\mathrm{Zn}\left(\mathrm{ClO}_{4}\right)_{2}$ only, since $\mathrm{PI}-\mathrm{ZnCl}_{2}$ is too fluid for tensile tests (Figure 3a, Table S4). The stress-strain curve of the monotonic loading shows that PI-Zn( $\left.\mathrm{ClO}_{4}\right)_{2}$ goes through a short linear elastic region followed by a long softening regime. The Young's modulus of 
(a)

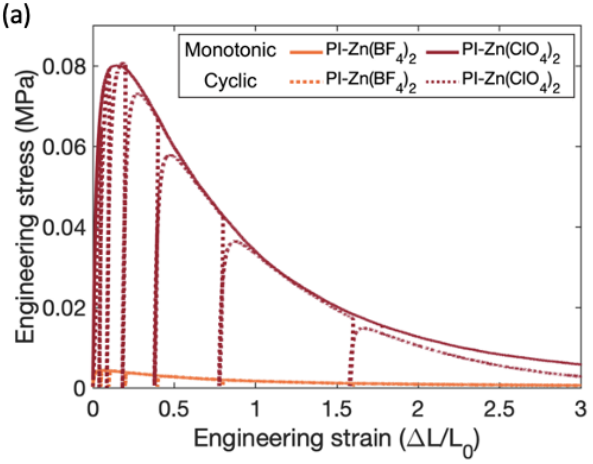

(c)

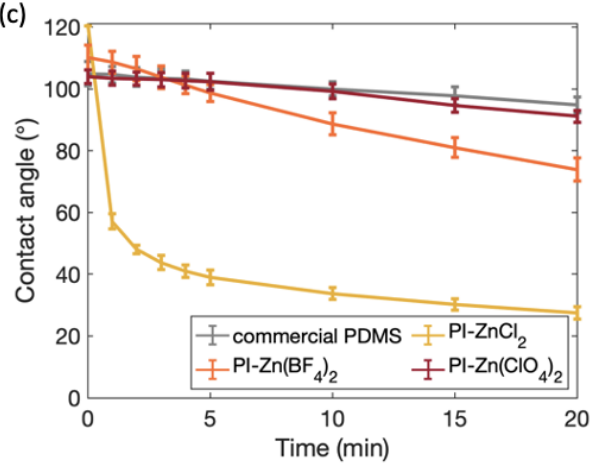

(e)

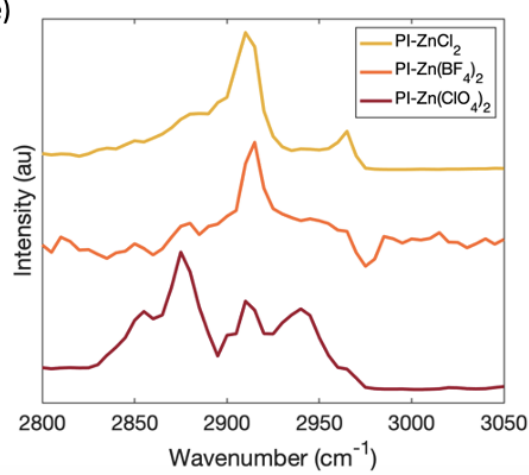

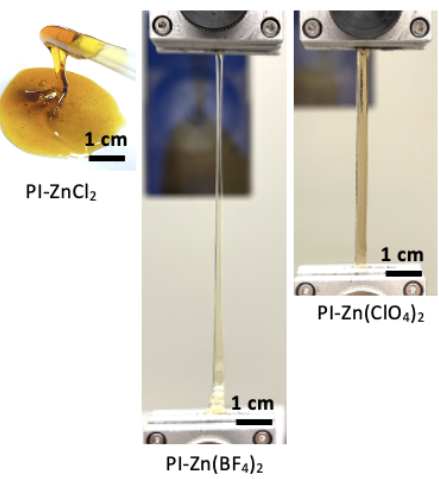

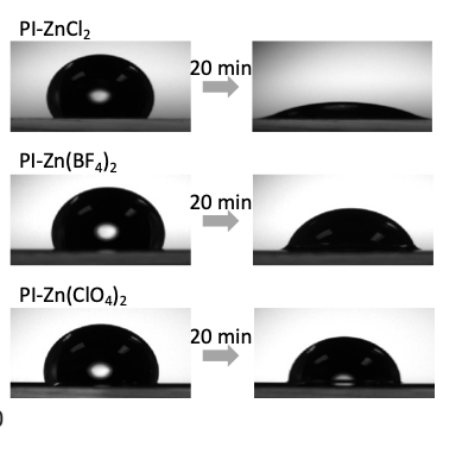

(f)

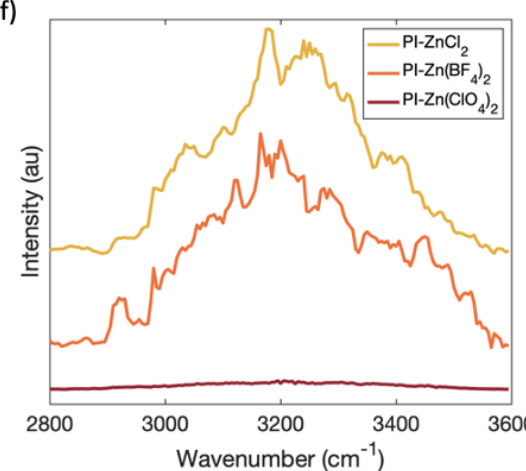

(d)
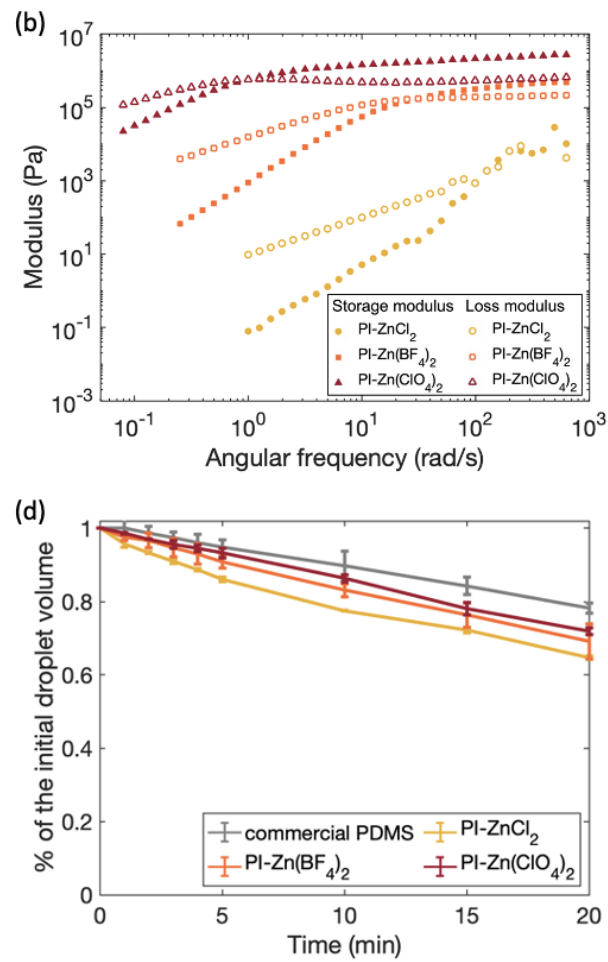

(g)

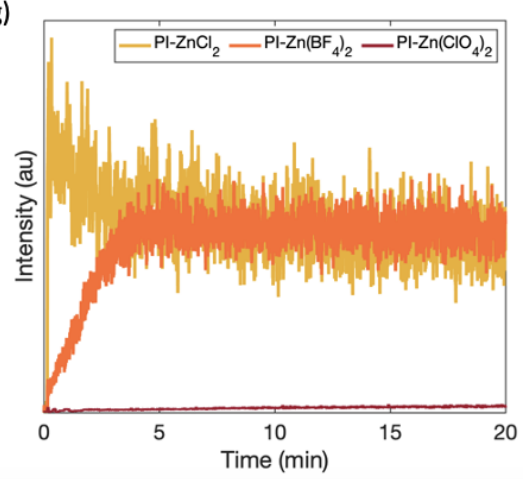

Figure 3: Comparison of the $\mathrm{Zn}(\mathrm{II})$-coordinated PI with different counter anions. (a) Monotonic and cyclic tensile tests plotted in engineering stress as a function of engineering strain and photos of the specimens. (b) Rheological plot of the different materials. (c) Change of the water contact angle on different PDMS surfaces and photos of the interface upon droplet attachment and after 20 min. (d) Change of the droplet volume. (e) SFG spectra in air. (f) SFG spectra in water after 20 min. (g) Time-dependent SFG signal intensity at $3200 \mathrm{~cm}^{-1}$.

$\mathrm{PI}-\mathrm{Zn}\left(\mathrm{ClO}_{4}\right)_{2}$ is about 13 times larger than $\mathrm{PI}-\mathrm{Zn}\left(\mathrm{BF}_{4}\right)_{2}$. The coordination strength between $\mathrm{Zn}(\mathrm{II})$ and pyridyl imine ligand under different counter anions can be identified from the stiffness of the materials: $\mathrm{Cl}^{-}$generates the weakest coordination and yields the weakest network, $\mathrm{BF}_{4}^{-}$is intermediate, and $\mathrm{ClO}_{4}^{-}$promotes the strongest coordination and enables the strongest network. Interestingly, although $\mathrm{PI}-\mathrm{Zn}\left(\mathrm{ClO}_{4}\right)_{2}$ has a much higher elastic modulus than PI-Zn( $\left(\mathrm{BF}_{4}\right)_{2}$, still almost no strain recovery is observed for $\mathrm{PI}-\mathrm{Zn}\left(\mathrm{ClO}_{4}\right)_{2}$ in the unloading process, indicating that the coordination between $\mathrm{Zn}$ (II) and pyridyl imine ligand with the presence of $\mathrm{ClO}_{4}^{-}$anions is strong yet labile, with the network retaining no memory of its initial configuration. To quantify the coordination lifetime, we performed rheology measurements (Figure 3b, Table S5). Since PI is free from entanglement, the relaxation of the dynamic network is governed by breaking and reforming the reversible crosslinks between $\mathrm{Zn}$ (II) cations and the pyridyl imine ligands. The characteristic relaxation time of the material $\left(\tau_{c}\right)$, which is equivalent to the coordination lifetime in this case, can be extracted from the reciprocal of angular frequency where the curves of storage modulus and loss modulus crossover each other.[58] As expected, $\mathrm{PI}_{-} \mathrm{ZnCl}_{2}$ has the shortest relaxation time, corresponding to a coordination lifetime of $\sim 0.03 \mathrm{~s}$. PI- $\mathrm{Zn}\left(\mathrm{BF}_{4}\right)_{2}$ has the intermediate relaxation time, corresponding to a coordination lifetime of $\sim 0.24 \mathrm{~s}$. $\mathrm{PI}-\mathrm{Zn}\left(\mathrm{ClO}_{4}\right)_{2}$ has the 
longest relaxation time, corresponding to a coordination lifetime of $\sim 6.31 \mathrm{~s}$.

The hydrophilicity of PI-ZnCl 2 and PI-Zn( $\left(\mathrm{ClO}_{4}\right)_{2}$ was measured by the tensiometer using the sessile drop method. From the wetting behavior of the water droplet on different materials, we see a clear contrast among the three $\mathrm{Zn}(\mathrm{II})$-coordinated PI. The contact angle of the $\mathrm{PI}-\mathrm{ZnCl}_{2}$ starts at $\sim 120^{\circ}$, indicating the surface is highly hydrophobic at the beginning, followed by a rapid drop to $\sim 40^{\circ}$ after $5 \mathrm{~min}$, and the contact angle keeps decreasing slowly afterwards (Figure 3c, Table S6). This transformation suggests that $\mathrm{PI}-\mathrm{ZnCl}_{2}$ has a fast-evolving surface, which exposes the polar functionality rapidly and interacts with water strongly, exhibiting high hydrophilicity. It can be clearly seen from the pictures that the water droplet on the $\mathrm{PI}-\mathrm{ZnCl}_{2}$ surface flattens out after $20 \mathrm{~min}$. In contrast, the contact angle of PI-Zn $\left(\mathrm{ClO}_{4}\right)_{2}$ starts at $\sim 104^{\circ}$ and decreases $\sim 13^{\circ}$ over $20 \mathrm{~min}$, exhibiting the lowest hydrophilicity among the three $\mathrm{Zn}(\mathrm{II})$-coordinated PI. Furthermore, the droplet volume decreases faster on the three Zn(II)-coordinated PI than the commercial PDMS (Figure 3d, Table S7), suggesting that water adsorption happens on the interfaces. The amount of water adsorbed is positively correlated with surface hydrophilicity: a more hydrophilic surface tends to adsorb more water.[59] From the above discussion, we can see that the weaker and more labile metal-ligand coordination enables a more dynamic PDMS network, which exposes the polar functionality more rapidly, and becomes more hydrophilic. SFG was used to study the interfacial behavior of PI-ZnCl 2 and PI-Zn( $\left(\mathrm{ClO}_{4}\right)_{2}$. SFG spectra were collected from the PDMS samples in air and water (Figure 3e, Figure 3f, and Figure S7). These spectra collected in air are different from each other due to the different surface structures of the three PDMS materials in air caused by the varied anions: $\mathrm{Cl}^{-}, \mathrm{BF}_{4}^{-}$, and $\mathrm{ClO}_{4}^{-}$. The $\mathrm{SFG}$ spectrum collected from the PI- $\mathrm{ZnCl}_{2}$ surface in air is dominated by a strong peak centered at around $2910 \mathrm{~cm}^{-1}$, along with a weak peak at around $2960 \mathrm{~cm}^{-1}$, contributed by the symmetric and asymmetric $\mathrm{C}-\mathrm{H}$ stretching modes of the $\mathrm{Si}_{-} \mathrm{CH}_{3}$ groups, similar to that collected from PI-Zn(BF $\left.\mathrm{BF}_{4}\right)_{2}$ in air. The strong SFG signals indicate that the surface $\mathrm{Si}-\mathrm{CH}_{3}$ groups are very ordered. This shows that the $\mathrm{PI}-\mathrm{ZnCl}_{2}$ surface is covered by the ordered hydrophobic Si-CH 3 groups in air, similar to the PI- $\mathrm{Zn}\left(\mathrm{BF}_{4}\right)_{2}$ surface. As discussed above, $\mathrm{Cl}^{-}$generates the weakest coordination among the three anions and yields the most dynamic PDMS network. SFG spectra collected from the PI- $\mathrm{Zn}\left(\mathrm{ClO}_{4}\right)_{2}$ surface in air is different from those detected from PI-ZnCl $\mathrm{Zn}_{2}$ and $\mathrm{PI}-\mathrm{Zn}\left(\mathrm{BF}_{4}\right)_{2}$. In addition to the peaks contributed by the $\mathrm{Si}_{-} \mathrm{CH}_{3}$ groups, SFG signals (e.g., at $2860 \mathrm{~cm}^{-1}$ and $2880 \mathrm{~cm}^{-1}$ ) are generated from other groups, such as various $\mathrm{CH}_{2}$ groups including $\mathrm{Si}-\mathrm{CH}_{2}, \mathrm{C}-\mathrm{CH}_{2}$, and $\mathrm{N}-\mathrm{CH}_{2}$ groups. Even though air is very hydrophobic, due to the suppressed dynamics of the material, the surface cannot be fully covered by the $\mathrm{Si}_{-} \mathrm{CH}_{3}$ groups. The SFG spectra in water show that the interfacial water molecules have greatly different interfacial orderings for the surfaces of the three materials. The PI- $\mathrm{ZnCl}_{2} /$ water interface generates the strongest $\mathrm{SFG}$ water signal with the highest ordering, followed by PI- $\mathrm{Zn}\left(\mathrm{BF}_{4}\right)_{2}$ and then $\mathrm{PI}-\mathrm{Zn}\left(\mathrm{ClO}_{4}\right)_{2}$. As expected, the three surfaces exhibit markedly different time-dependent changes in water (Figure 3g and Figure S8). The SFG signal collected from the PI-ZnCl 2 /water interface increased immediately after water contact, then decreased over time to $\sim 1 / 2$ of the maximum; this overshoot was not observed for any of the less dynamic networks. The overshoot for $\mathrm{PI}-\mathrm{ZnCl}_{2}$ is likely due to the strong immediate water interaction as the hydrophilic metal-binding moieties move to the surface in response to water contact, followed by a slower adjustment as water is adsorbed into the material. The time-dependent SFG signal intensity change detected from the PI- $\mathrm{Zn}\left(\mathrm{ClO}_{4}\right)_{2}$ /water interfaces is the slowest of the three counter anions as expected from the longest crossover time in the rheological measurements, and the smallest contact angle change. The above presented SFG studies on the three PDMS materials in air, in water, and time-dependent surface structural changes in water provide molecular level interpretation on the above contact angle measurement results. Clearly, the different surface behavior of the three PDMS materials is caused by the different network dynamics as tuned by the choice of counter anion.

Based on what we have learned from the experimental results, a wetting mechanism of a water droplet on the dynamic metal-ligand coordinated PDMS surface is proposed to explain the contact angle decrease and the hydrophilicity increase of the surface (Figure 4). Prior to the addition of a water droplet, the metal-ligand coordinated PDMS behaves like regular PDMS. The PDMS backbones tend to cover up the surface and the metal-ligand coordination sites lie underneath. This configuration is energetically 

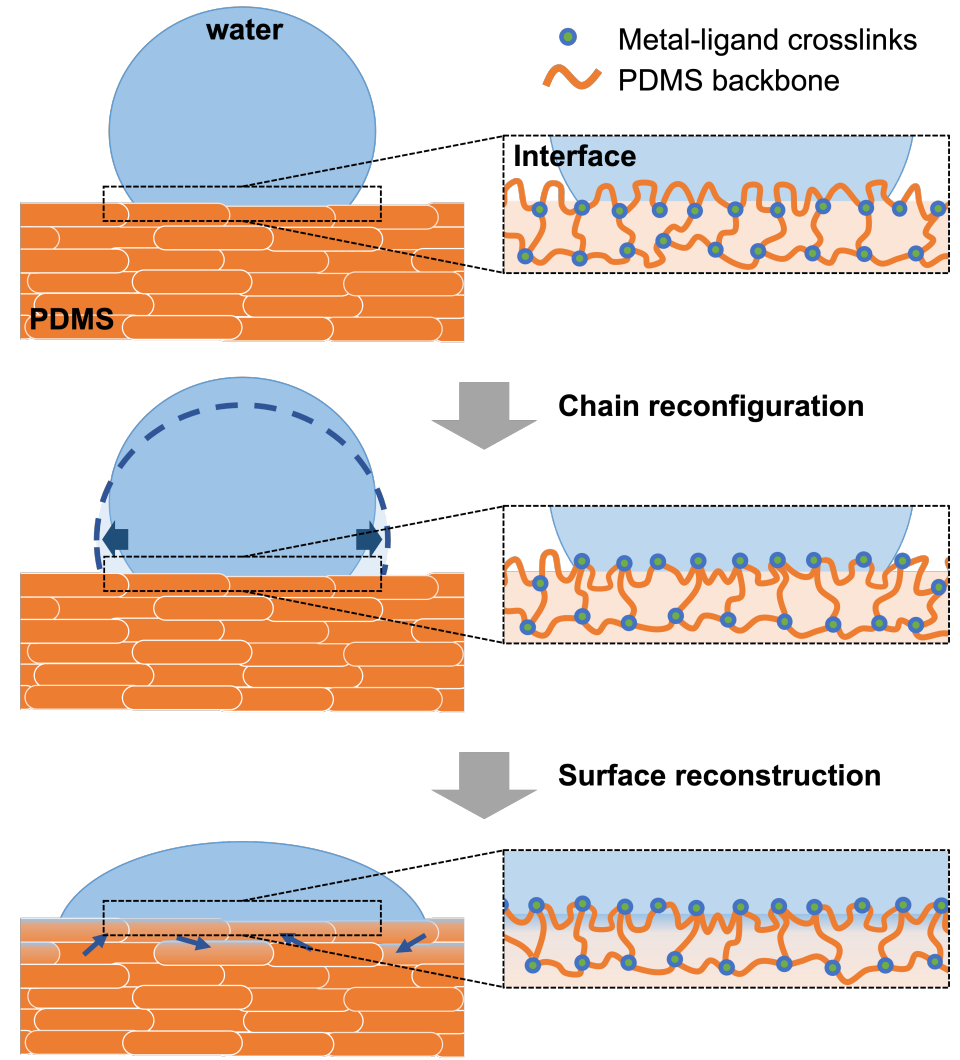

Figure 4: Schematic of the wetting mechanism of a sessile drop on the metal-ligand coordinated PDMS surface.

favorable because the non-polar $\left[\mathrm{SiO}\left(\mathrm{CH}_{3}\right)_{2}\right]$ backbone has lower surface energy than the polar metalligand sites.[60] Consequently, the initial contact angles measured for the metal-ligand coordinated PDMS are within the range of the conventional PDMS. When the droplet touches the surface, the hydrophilic metal-ligand coordination sites tend to interact with the water, whereas the hydrophobic PDMS backbone segments tend to repel the water. In a dynamic network, chain reconfiguration can occur. Since the interfacial energy is lowered in the new chain arrangement, the droplet tends to increase the contact area with the surface, so the contact line moves outwards, which triggers chain reconfiguration in the newly wetted area and further lowers the interfacial energy.[61] The wetting process continues, resulting in an elongating contact line and decreasing contact angle. In the meantime, water adsorption happens on the interface due to the strong interaction between the metal-ligand coordination sites and the water molecules. Due to the high mobility, some chains from the bulk migrate to the upper surface gradually and further interact with water. This surface reconstruction process leads to more water adsorption on the surface. On the contrary, if the network is fully constrained or simply less dynamic, chain reconfiguration will be slower, so the wetting resulting from the polarity increase will be too slow to be observed in a relatively short time period, and instead, evaporation dominates the contact angle change of the droplet.[52]

\subsection{Influence of Metal Cations}

Moving forward, we demonstrate another building block, the metal cation, and its influence on the PDMS hydrophilicity. PI-Fe( $\left(\mathrm{BF}_{4}\right)_{2}$ and PI-Co(BF 4$)_{2}$ were synthesized, and the coordinated structure was confirmed by FTIR (Figure S9). The variation in network dynamics resulting from incorporating different metal cations is characterized by mechanical testing. The stress-strain curves show that the elastic modulus significantly increases from $\mathrm{Zn}$ (II) to $\mathrm{Fe}(\mathrm{II})$ to $\mathrm{Co}(\mathrm{II})$, indicating that the coordination strength has the order of $\mathrm{Zn}(\mathrm{II})<\mathrm{Fe}(\mathrm{II})<\mathrm{Co}(\mathrm{II})$ (Figure 5a, Table S8). Both PI-Fe(BF 4$)_{2}$ and $\mathrm{PI}-\mathrm{Co}\left(\mathrm{BF}_{4}\right)_{2}$ show clear strain recovery in the unloading process, but PI-Fe $\left(\mathrm{BF}_{4}\right)_{2}$ recovers less than PI-Co $\left(\mathrm{BF}_{4}\right)_{2}$ and leaves a more pronounced hysteresis, suggesting that the metal-ligand bond kinetics has the order of $\mathrm{Zn}(\mathrm{II})>$ 

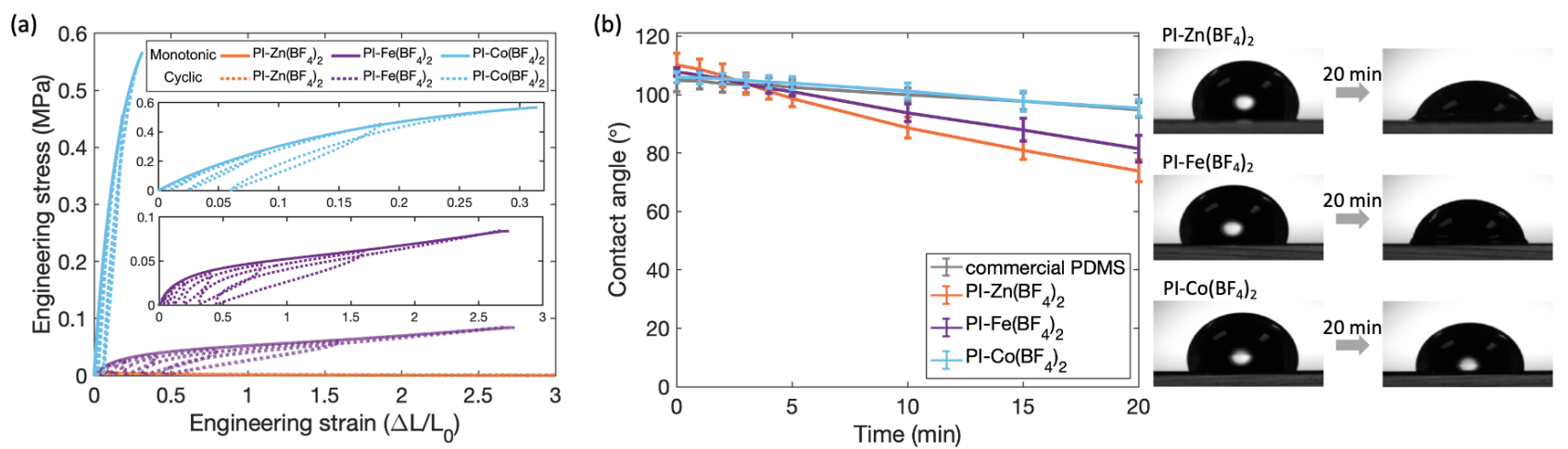

Figure 5: Comparison of different metal cation coordinated PI. (a) Monotonic and cyclic tensile tests plotted in engineering stress as a function of engineering strain. (b) Change of the water contact angle on different PDMS surfaces and photos of the interface upon droplet attachment and after $20 \mathrm{~min}$.

$\mathrm{Fe}(\mathrm{II})>\mathrm{Co}(\mathrm{II})$. Therefore, with the same counter anion, $\mathrm{Zn}$ (II) generates the most dynamic network among the three metal cations, Fe(II) is the intermediate, and $\mathrm{Co}$ (II) yields the least dynamic network. Moreover, the difference in PDMS hydrophilicity resulting from incorporating different metal cations can be observed from the wetting behavior of water droplets. PI-Fe( $\left.\mathrm{BF}_{4}\right)_{2}$ shows faster contact angle decrease than PI-Co(BF $)_{2}$, but still less dramatic than PI-Zn(BF $)_{2}$ (Figure 5b, Table S9). Both PI$\mathrm{Fe}\left(\mathrm{BF}_{4}\right)_{2}$ and $\mathrm{PI}-\mathrm{Co}\left(\mathrm{BF}_{4}\right)_{2}$ show water adsorption on the surface, but less than $\mathrm{PI}-\mathrm{Zn}\left(\mathrm{BF}_{4}\right)_{2}$ (Figure S10, Table S10).

To examine the long time wetting behavior on different metal coordinated PDMS surfaces, we placed the PDMS coated glass slides in a chamber to maintain humidity, and measured the contact angle change after 24 hrs (Figure S14). The contact angle of PI-Fe(BF $)_{2}$ drops to $\sim 34^{\circ}$ from the initial value, and the contact angle of PI-Co(BF 4$)_{2}$ drops to $\sim 62^{\circ}$ from the initial value (Table S11). These results again confirm that the hydrophilicity of the metal-ligand coordinated PDMS network is directly correlated to the network dynamics: among the three metal cations compared, PI-Zn( $\left.\mathrm{BF}_{4}\right)_{2}$ yields the most dynamic network, and therefore exhibits the highest hydrophilicity and the fastest evolution over time; whereas PI-Co( $\left(\mathrm{BF}_{4}\right)_{2}$ yields the least dynamic network, and therefore exhibits the lowest hydrophilicity and the slowest evolution.

\subsection{Application in Marine Antifouling Coatings}

One potential application for metal-ligand coordinated PDMS is as a marine fouling-release coating. PDMS is a good candidate for fouling-release coatings due to its low surface energy that promotes weak adhesion of marine organisms. Therefore, the organisms settled on the surface can be easily removed under hydrodynamic shear stresses produced by water during ship movement or water jet cleaning. [6, 14] We used diatoms as a fouling species to examine the fouling release properties of the metal-ligand coordinated PDMS. PI-Co( $\left(\mathrm{BF}_{4}\right)_{2}$ was selected as the coating material considering the slow-release hydrophilicity introduced by the $\mathrm{Co}$ (II) coordination and the long term stability of the polymer under water. DIP$\mathrm{Co}\left(\mathrm{BF}_{4}\right)_{2}$ and commercial PDMS (Dow Corning ${ }^{\circledR}$ 3-0213) were used as controls. To evaluate the antifouling behavior, standard diatom adhesion and removal assays were carried out (Figure 6a). The density of cells attached to the surface was counted using a Leica LAS X image analysis system attached to a Zeiss Axioscop fluorescence microscope (Figure 6b). Initial attachment densities on the Co(II)-coordinated PDMS coatings and the commercial PDMS coatings were broadly similar (Figure 6c), reflecting similar tendency of cells to attach firmly to the three surfaces. Interestingly, $\mathrm{PI}-\mathrm{Co}\left(\mathrm{BF}_{4}\right)_{2}$ exhibits a considerably higher removal rate than the controls subject to a hydrodynamic shear stress of $38 \mathrm{~Pa}$ in a calibrated water channel.[62] As a result, the density of remaining diatoms is the lowest on the $\mathrm{PI}-\mathrm{Co}(\mathrm{BF} 4)_{2}$ coatings $(\sim 1 / 3$ of the controls) confirming its superior fouling-release properties (Figure $6 \mathrm{c}$ ). We attribute this result to the progressively developed hydrophilicity of $\mathrm{PI}-\mathrm{Co}\left(\mathrm{BF}_{4}\right)_{2}$ weakening the attachment of diatoms, which facilitates their detachment during hydrodynamic washing. The SFG spectra 


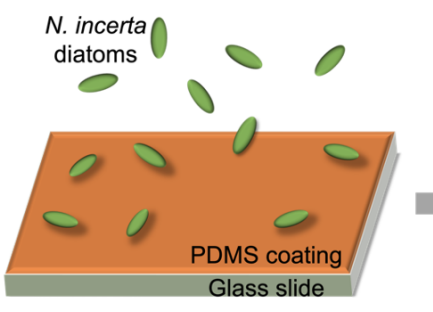

Diatoms land on surface by gravity

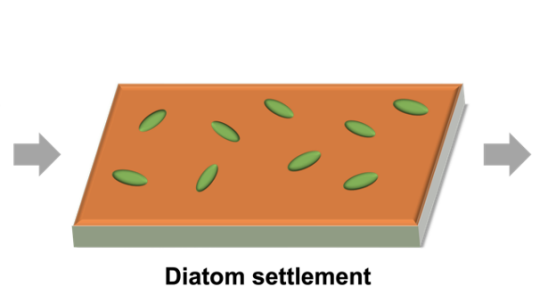

Diatom settlement

(c) (b)

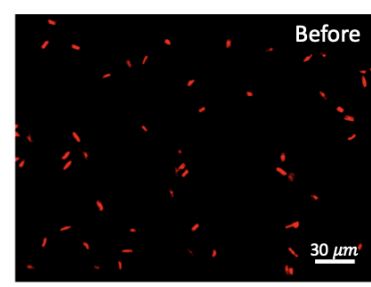

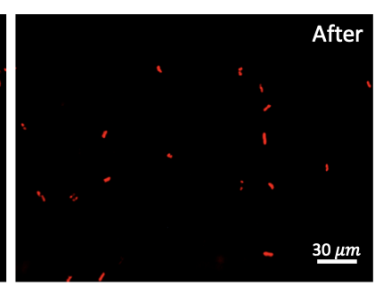

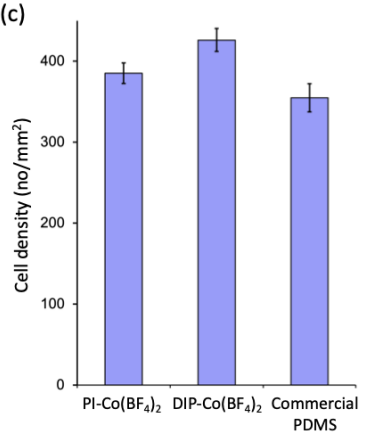

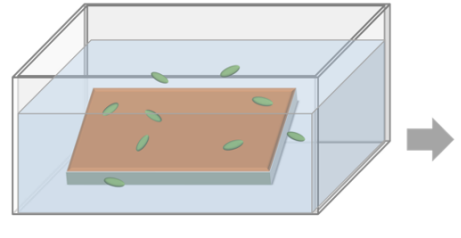

Expose to a shear stress in a water channel

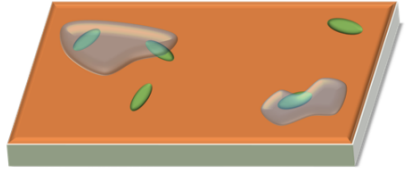

Examine fouling-release result
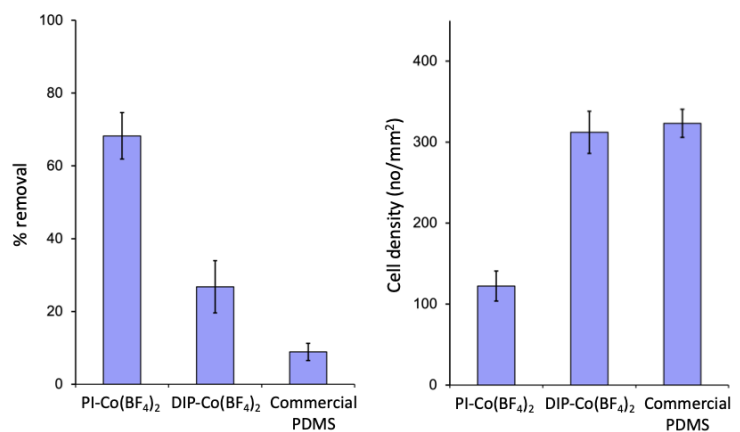

Figure 6: (a) Schematic of the diatom fouling test procedures. (b) Images of typical diatom cell densities on a PI$\mathrm{Co}\left(\mathrm{BF}_{4}\right)_{2}$ coating before and after exposure to a shear stress. Cells have been fixed and dried and imaged using fluorescence microscopy. (c) Results of PI-Co(BF $)_{2}$ coatings in the diatom fouling test compared to the controls, DIP-Co(BF $)_{2}$ and commercial PDMS. Left: the density of attached diatoms on the coatings after 2 hours. Middle: percent removal of diatoms. Right: the density of diatoms remaining on the coatings after the removal process. Each data is the mean from 90 counts from three replicate slides. Bars show $95 \%$ confidence limits.

confirm that the water ordering on the surface of PI-Co( $\left(\mathrm{BF}_{4}\right)_{2}$ is much greater than that of $\mathrm{DIP}-\mathrm{Co}(\mathrm{BF})_{2}$ (Figure S16).

\section{Conclusion}

In this manuscript, polar functionalities consisting of metal-ligand coordination were introduced into PDMS as a innovative method to tune the hydrophilicity of the material. The time frame and degree of influence of the metal salt is set by the network dynamics via metal-ligand coordination. We first demonstrated the idea using the pyridyl imine ligand functionalized PDMS, along with the controls consisting of the diiminopyridine ligand functionalized PDMS and commercial PDMS. Both ligand functionalized PDMS were coordinated by the same metal salt $\left(\mathrm{Zn}\left(\mathrm{BF}_{4}\right)_{2}\right)$, and the difference in the network dynamics was carefully investigated by the monotonic and cyclic loading tests: the model system exhibits more dynamic mechanical behavior than the control. The hydrophilicity of the materials was monitored by the water contact angle: the model system exhibits improved hydrophilicity upon exposure to water, whereas the controls show no hydrophilicity change. The interfacial molecular behavior studied by SFG shows that in air the hydrophobic $\mathrm{Si}-\mathrm{CH}_{3}$ groups in a dynamic PDMS network can segregate to cover almost the entire surface with an ordered structure. When the material is in contact with water, the surface reconstructs and bonds water molecules at the interface. The results confirm that the material hydrophilicity is dominated by the dynamic nature of the network: fast chain motion facilitates the exposure of polar metal-ligand sites, which interact strongly with water, and thus the material exhibits increased hydrophilicity over time. Informed by these findings, we further manipulated the dynamics of the Zn(II)-pyridyl imine coordinated PDMS by varying the counter anions. The mechanical and rheological characterizations, contact angle results, together with SFG studies again confirm that dynamic coordination promotes the exposure of polar functionalities and thus enables strong surface interactions with water. We discussed the wetting mechanism of a sessile water droplet on the dynamic metal-ligand coordinated PDMS surface. The observed contact angle progression and water adsorption were explained in the context of chain reconfiguration and surface reconstruction, which are dominated by the dynamics 
of the network. We also show that the PDMS surface hydrophilicity could be tuned by other design factors, such as altering the metal cations in metal-ligand coordination. Lastly, we performed diatom fouling tests on the metal-coordinated PDMS, which shows superior fouling-release properties compared to the controls. The insights gained from this work could potentially improve the application of PDMS in various fields.

\section{Experimental Section}

Materials: Aminopropyl terminated polydimethylsiloxane $\left(\mathrm{H}_{2} \mathrm{~N}-\mathrm{PDMS}-\mathrm{N}_{2} \mathrm{H}\right)$ with the viscosity of $20-$ $30 c S t$ was purchased from Gelest. 2-pyridinecarboxaldehyde, zinc(II) tetrafluoroborate hydrate, zinc(II) perchlorate hexahydrate, iron(II) tetrafluoroborate hexahydrate, chloroform, toluene, dichloromethane, methanol, tetrahydrofuran and molecular sieves 3A were purchased from MilliporeSigma. 2,6-pyridinedicarboxaldehyde was purchased from TCI Chemical. Zinc(II) chloride hydrate and cobalt(II) tetrafluoroborate hydrate were purchased from Alfa Aesar. Hexane and acetonitrile were purchased from Fisher Chemical. P-toluenesulfonic acid monohydrate were purchased from Oakwood Chemical. Toluene and chloroform were dried by activated 3A molecular sieves. All other materials were used as received.

Synthesis of pyridyl imine functionalized PDMS: PI was synthesized via a condensation reaction. The method was from a previously published procedure.[41] $\mathrm{H}_{2} \mathrm{~N}-\mathrm{PDMS}-\mathrm{N}_{2} \mathrm{H}$ with the viscosity of $20-30 c S t$ $(50 \mathrm{~g}, 0.03 \mathrm{~mol})$ was dissolved in chloroform $(100 \mathrm{ml})$, and 2-pyridinecarboxaldehyde $(6.6 \mathrm{~g}, 0.06 \mathrm{~mol})$ was then added into the solution. 3A molecular sieves $(200 \mathrm{~g})$ was used to absorb the water generated during the reaction. The mixture was stirred at room temperature for $48 \mathrm{hr}$. Chloroform was then removed under rotary evaporation. The product was redissolved in hexane $(50 \mathrm{ml})$ and excess 2-pyridinecarboxaldehyde was extracted by acetonitrile $(100 \mathrm{ml})$. The PDMS product was dried by rotary evaporation, and then left in a vacuum oven at $50{ }^{\circ} \mathrm{C}$ for $12 \mathrm{hr}$ to completely remove the solvent. The final product was filtered through a $0.45 \mu \mathrm{m}$ PTFE filter, obtaining yellow colored PDMS oil (46 $g$, yield 81\%). ${ }^{1} \mathrm{H} \mathrm{NMR}\left(500 \mathrm{MHz}, \mathrm{CDCl}_{3}\right) \delta 8.64(\mathrm{ddd}, J=4.9,1.8,1.1 \mathrm{~Hz}, 2 \mathrm{H}), 8.37(\mathrm{t}, J=1.6 \mathrm{~Hz}, 2 \mathrm{H})$, $7.99(\mathrm{dt}, J=7.9,1.1 \mathrm{~Hz}, 2 \mathrm{H}), 7.73(\mathrm{td}, J=7.7,1.9 \mathrm{~Hz}, 2 \mathrm{H}), 7.30(\mathrm{ddd}, J=7.6,4.9,1.4 \mathrm{~Hz}, 2 \mathrm{H}), 3.66$ $(\mathrm{td}, J=7.0,1.4 \mathrm{~Hz}, 2 \mathrm{H}), 1.82-1.71(\mathrm{~m}, 4 \mathrm{H}), 0.65-0.50(\mathrm{~m}, 4 \mathrm{H}), 0.90-0.03(\mathrm{~m}, 155 \mathrm{H})$.

Synthesis of diiminopyridine functionalized PDMS: DIP was synthesized according to a previously reported procedure.[44] $\mathrm{H}_{2} \mathrm{~N}-\mathrm{PDMS}-\mathrm{N}_{2} \mathrm{H}$ with the viscosity of $20-30 \mathrm{cSt}(50 \mathrm{~g}, 0.03 \mathrm{~mol})$ was dissolved in toluene $(100 \mathrm{ml})$. 2,6-pyridinedicarboxaldehyde $(4 \mathrm{~g}, 0.03 \mathrm{~mol})$ and p-toluenesulfonic acid monohydrate $\left(53 \mathrm{mg}, 0.3 \mathrm{mmol}\right.$ ) were added into the solution. The mixture was heated to $120{ }^{\circ} \mathrm{C}$ in an oil bath and stirred for $10 \mathrm{hr}$ under an argon atmosphere. When the reaction finished, the solution was cooled down to room temperature and the toluene was removed by rotary evaporation. The product was then dissolved in dichloromethane $(50 \mathrm{ml})$ and transferred into a separatory funnel. Methanol $(50 \mathrm{ml})$ was then added to extract the excess 2,6-pyridinedicarboxaldehyde. The mixture was settled to phase separate and the PDMS phase was collected. The product was dried under vacuum to completely remove the solvent, obtaining deep orange colored viscous PDMS oil (35 g, yield $65 \%)$. ${ }^{1} \mathrm{H}$ NMR $(500 \mathrm{MHz}$, $\left.\mathrm{CDCl}_{3}\right) \delta 8.40(\mathrm{~s}, 2 \mathrm{H}), 8.01(\mathrm{~d}, J=7.3 \mathrm{~Hz}, 2 \mathrm{H}), 7.78(\mathrm{t}, J=7.8 \mathrm{~Hz}, 1 \mathrm{H}), 3.67(\mathrm{t}, J=6.9 \mathrm{~Hz}, 4 \mathrm{H})$, $1.76(\mathrm{dt}, J=15.6,7.9 \mathrm{~Hz}, 4 \mathrm{H}), 0.65-0.56(\mathrm{~m}, 4 \mathrm{H}), 0.09-0.03(\mathrm{~m}, 166 \mathrm{H})$.

Synthesis of metal-coordinated complexes: $2 \mathrm{~g}$ ligand functionalized PDMS was dissolved in $20 \mathrm{ml}$ THF, and a certain amount of metal salt calculated from stoichiometry was dissolved in THF $(0.1 \mathrm{~g} / \mathrm{ml})$ in a separate vial. The metal salt solution was added dropwise into the PDMS solution, and the mixture was stirred until homogeneous. The solution was then concentrated to $5 \mathrm{ml}$ and poured into a Teflon mold or drop cast on a glass slide for the contact angle test. The polymer was dried at ambient atmosphere overnight, and then dried in the vacuum oven at $50{ }^{\circ} \mathrm{C}$ for $24 \mathrm{hr}$.

${ }^{1} H$ nuclear magnetic resonance $\left({ }^{1} H N M R\right)$ : The ${ }^{1} \mathrm{H}$ NMR spectrum of the linear polymer was acquired on a Bruker Advance III HD 500 spectrometer. The polymer was dissolved in $\mathrm{CDCl}_{3}$ and processed by 16 scans, with $30 s$ relaxation delay and $90^{\circ}$ excitation pulse. 
Gel permeation chromatography $(G P C)$ : The molecular weight and polydispersity of PI and DIP were conducted on a Waters Ambient Temperature GPC equipped with triple detectors. THF was used as the eluent with a flow rate of $1.00 \mathrm{~mL} / \mathrm{min}$. Monodispersed polystyrene was used as standards. The GPC sample was prepared by dissolving the polymer in THF with a concentration of $1 \mathrm{mg} / \mathrm{mL}$ and then filtering through a $0.45 \mu \mathrm{m}$ PTFE filter.

Contact angle tests: All the contact angle measurements were conducted on an Attension Theta Lite tensiometer. Sessile-drop goniometry was used in each measurement. Glass slides coated with polymer were placed horizontally on the platform and a droplet of water or other measuring liquid was placed on the surface. The droplet profile was recorded by the camera for $10 \mathrm{~s}$, and the contact angle at the interface between the substrate and the liquid was analyzed by the software automatically. Each sample was measured multiple times $(>3)$ at different locations, and the contact angle was the statistical average of the measured values.

Mechanical tests: The monotonic and cyclic tensile tests were all carried out on a Zwick-Roell Z010 system with a $20 N$ capacity load cell. The monotonic tensile tests were performed under a constant engineering strain rate of $0.01 \mathrm{~s}^{-1}$. The cyclic tensile tests were performed under a constant engineering strain rate of $0.01 \mathrm{~s}^{-1}$ with the displacement control in the loading direction and the force control with a force of $0.001 N$ in the unloading direction. Samples were cut into a rectangular shape $(\mathrm{L} \times \mathrm{W} \times \mathrm{H} \approx$ $40 \mathrm{~mm} \times 4 \mathrm{~mm} \times 0.8 \mathrm{~mm}$ ), and the initial grip-to-grip separation was set to $20 \mathrm{~mm}$. Each material was measured 3 times.

Rheology tests: The rheology measurements were carried out on a TA Instruments DHR-3 rheometer using the $20 \mathrm{~mm}$-diameter parallel plate. A frequency sweep with $1 \%$ oscillatory strain was performed. All the samples were equilibrated at $25^{\circ} \mathrm{C}$ for $10 \mathrm{~min}$ before start.

Sum Frequency Generation (SFG) vibrational spectroscopy: SFG vibrational spectroscopy is a second order nonlinear optical spectroscopy.[33, 34, 35, 36, 37] SFG theories, equipment, and data analysis have been extensively reported.[33, 34, 35, 36, 37] In an SFG process, two photons of light interact with the same molecule, resulting in the release of a third photon with the combine energy-sum frequency-of the two input photons. Experimentally, a visible pulsed laser $(532 \mathrm{~nm})$ and a frequency tunable IR pulsed laser are overlapped spatially and temporally at the interface of interest, resulting in the generation of SFG signals from the interface. Here SFG is a vibrational spectroscopic process, meaning enhanced signals come from IR wavelengths that are in resonance with the vibrational modes of the materials being studied. This generates a vibrational spectrum of the materials. The selection rules of SFG are such that only materials without inversion symmetry can generate SFG signal (under the electric dipole approximation). This means that SFG signals will not be generated from bulk materials, as they are usually centrosymmetric and therefore have inversion symmetry. This means that only the interface between two materials where the inversion symmetry is broken will generate SFG signals. This makes SFG an inherently surface specific spectroscopic technique that allows for the study of interface in situ, nondestructively. This study used a commercial SFG spectrometer from EKSPLA and all the SFG spectra were collected using ssp (s-polarized sum frequency signal, s-polarized input green beam, and p-polarized IR beam) polarization combinations. [63, 64, 65] In this work, to collect SFG spectra, right-angle $\mathrm{CaF}_{2}$ prisms (Altos Photonics, Bozeman, MT) were used as substrates. PI and DIP materials used for SFG study were prepared by dissolving $1 \mathrm{~g}$ of a salt and $1 \mathrm{~g}$ of the appropriate PDMS in separate $5 \mathrm{~mL}$ aliquots tetrahydrofuron (THF). Once fully dissolved, the two aliquots of THF were mixed and vortexed at $5000 \mathrm{rpm}$ for $30 \mathrm{~min}$ to ensure thorough mixing of the solutions. The materials were then spin coated onto $\mathrm{CaF}_{2}$ prisms at $2000 \mathrm{rpm}$ for $60 \mathrm{sec}$. The coated prisms where then dried at $55^{\circ}$ for $24 \mathrm{hr}$. Before spin coating, $\mathrm{CaF}_{2}$ prisms were rinsed with detergent solution, water, ethanol, and toluene. They were then polished and plasma cleaned for $60 \mathrm{sec}$ to ensure no contaminants were on the surface. SFG spectra were collected from the metal PDMS surfaces with variable anion species, metal species, and ligands bound to the PDMS chains. The SFG spectra of these materials were collected in air in the C-H stretching frequency region of $2800-3100 \mathrm{~cm}^{-1}$ and in water in the combined $\mathrm{C}-\mathrm{H} / \mathrm{O}-\mathrm{H}$ stretching frequency regions, measuring from $2800-3600 \mathrm{~cm}^{-1}$. First, the SFG spectrum was collected in air to observe the 
molecular structure at the PDMS/air interface. Second, the intensity of the SFG signal at $3200 \mathrm{~cm}^{-1}$ of the PDMS surface while in contact with deionized water was measured for 20 min to observe interfacial water structural changes over time. Finally, the combined C-H/O-H stretching spectrum was measured with the PDMS material in contact with deionized water after the previous 20 min experiment to observe the surface-water interactions after the system had reached equilibrium. It is worth mentioning that the time-dependent SFG signal intensities were calibrated with the SFG spectra collected in water.

Diatom fouling tests: The metal-ligand coordinated PDMS was coated on glass slides cleaned by piranha solution, with 6 slides for each samples. All coatings were pre-immersed for $48 \mathrm{hr}$ in $0.22 \mu \mathrm{m}$ filtered artificial seawater prior to the assay in order to equilibrate. Cells of Navicula incerta were cultured in F/2 medium contained in $250 \mathrm{ml}$ conical flasks. After 3 days the cells were in log phase growth. Cells were washed 3 times in fresh medium before harvesting and diluted to give a suspension with a chlorophyll content of approximately $0.25 \mu \mathrm{g} / \mathrm{ml}$. For initial attachment, cells were settled on three replicate coated slides of each sample in individual quadriPERM ${ }^{\circledR}$ dishes containing $10 \mathrm{ml}$ of suspension at around $20{ }^{\circ} \mathrm{C}$ on the laboratory bench. After $2 \mathrm{hr}$ the slides were exposed to $5 \mathrm{~min}$ of shaking on an orbital shaker (60 rpm) followed by a submerged wash in seawater to remove cells that had not attached (the immersion process avoided passing the samples through the air-water interface). Samples were fixed in $2.5 \%$ glutaraldehyde, air dried and the density of cells attached to the surface was counted on each slide using a Leica LAS X image analysis system attached to a Zeiss Axioscop fluorescence microscope. Cells were visualised by autofluorescence of chlorophyll. Counts were made for 30 fields of view (each $0.15 \mathrm{~mm}^{2}$ ) on each slide. For diatoms removal, a further three slides of each coating were settled with cells of $N$. incerta as described above. Slides with attached cells were exposed to a shear stress of $42 \mathrm{~Pa}$ in a water channel for 5 min.[62] Samples were fixed and the number of cells remaining attached was counted as described above.

\section{Supporting Information}

Supporting Information is available from the Wiley Online Library or from the author.

\section{Acknowledgements}

The work was supported by the Office of Naval Research under Grants no. N00014-17-1-2989, N0001420-1-2234, and N00014-20-1-2248 under the administration of PO Dr. Armistead. This work made use of the Cornell Center for Materials Research Shared Facilities which are supported through the NSF MRSEC program (DMR-1719875).

\section{References}

[1] U. Eduok, O. Faye, J. Szpunar, Progress in Organic Coatings 2017, 111124.

[2] A. Gökaltun, Y. B. A. Kang, M. L. Yarmush, O. B. Usta, A. Asatekin, Scientific Reports 2019, 9, 1 1.

[3] J. Peng, A. P. Tomsia, L. Jiang, B. Z. Tang, Q. Cheng, Nature Communications 2021, $12,11$.

[4] H. Zhang, M. Chiao, Journal of Medical and Biological Engineering 2015, 35, 2143.

[5] C. Wang, C. Wang, Z. Huang, S. Xu, Advanced Materials 2018, 30, 501801368.

[6] A. K. Leonardi, C. K. Ober, Annual Review of Chemical and Biomolecular Engineering 2019, 10 241.

[7] R. Dong, Y. Liu, L. Mou, J. Deng, X. Jiang, Advanced Materials 2019, 31, 451805033.

[8] K. Raj M, S. Chakraborty, Journal of Applied Polymer Science 2020, 137, 2748958.

[9] Y. Liu, S. Cui, J. Wei, H. Li, J. Hu, S. Chen, Y. Chen, Y. Ma, S. Wang, X. Feng, Advanced Intelligent Systems 2021, 2100072. 
[10] D. Qi, K. Zhang, G. Tian, B. Jiang, Y. Huang, Advanced Materials 2021, 33, 62003155.

[11] D. Wang, B. Sheng, L. Peng, Y. Huang, Z. Ni, Polymers 2019, 11, 91433.

[12] W. Yang, C. Li, M. Wang, X. Yu, J. Fan, Y. Xiong, Y. Yang, L. Li, IEEE Sensors Journal 2020, 21, 151.

[13] A. M. Maan, A. H. Hofman, W. M. de Vos, M. Kamperman, Advanced Functional Materials 2020, 30, 322000936.

[14] P. Hu, Q. Xie, C. Ma, G. Zhang, Langmuir 2020, 36, 92170.

[15] S. Vudayagiri, M. D. Junker, A. L. Skov, Polymer Journal 2013, 45, 8871.

[16] D. Fuard, T. Tzvetkova-Chevolleau, S. Decossas, P. Tracqui, P. Schiavone, Microelectronic Engineering 2008, 85, 5-6 1289.

[17] A. Mata, A. J. Fleischman, S. Roy, Biomedical Microdevices 2005, 7, 4281.

[18] S. Bhattacharya, A. Datta, J. M. Berg, S. Gangopadhyay, Journal of Microelectromechanical Systems 2005, 14, 3590.

[19] K. Efimenko, W. E. Wallace, J. Genzer, Journal of Colloid and Interface Science 2002, $254,2306$.

[20] D. Bodas, C. Khan-Malek, Sensors and Actuators B: Chemical 2007, 123, 1368.

[21] B. Gong, J. C. Spagnola, G. N. Parsons, Journal of Vacuum Science 6 Technology A: Vacuum, Surfaces, and Films 2012, 30, 1 01A156.

[22] H.-Y. Chen, J. Lahann, Analytical Chemistry 2005, 77, 216909.

[23] V. Barbier, M. Tatoulian, H. Li, F. Arefi-Khonsari, A. Ajdari, P. Tabeling, Langmuir 2006, 22, 12 5230 .

[24] S. Hu, X. Ren, M. Bachman, C. E. Sims, G. Li, N. Allbritton, Analytical Chemistry 2002, 74, 16 4117.

[25] M. Yao, J. Fang, Journal of Micromechanics and Microengineering 2012, 22, 2025012.

[26] A. Rahimi, S. J. Stafslien, L. Vanderwal, J. A. Finlay, A. S. Clare, D. C. Webster, Progress in Organic Coatings 2020, 149105931.

[27] M. E. Barry, E. C. Davidson, C. Zhang, A. L. Patterson, B. Yu, A. K. Leonardi, N. Duzen, K. Malaviya, J. L. Clarke, J. A. Finlay, et al., Macromolecules 2019, 52, 31287.

[28] K.-Y. Law, The Journal of Physical Chemistry Letters 2014, 5, 4 686-688.

[29] D. K. Owens, R. Wendt, Journal of Applied Polymer Science 1969, 13, 81741.

[30] M. Lejars, A. Margaillan, C. Bressy, Chemical Reviews 2012, 112, 84347.

[31] E. Khare, N. Holten-Andersen, M. J. Buehler, Nature Reviews Materials 2021, 6, 5421.

[32] S. C. Grindy, R. Learsch, D. Mozhdehi, J. Cheng, D. G. Barrett, Z. Guan, P. B. Messersmith, N. Holten-Andersen, Nature Materials 2015, 14, 121210.

[33] Y. Shen, Nature 1989, 337, 6207519.

[34] Z. Chen, Y. Shen, G. A. Somorjai, Annual Review of Physical Chemistry 2002, 53, 1437.

[35] G. Richmond, Chemical Reviews 2002, 102, 82693.

[36] X. Lu, C. Zhang, N. Ulrich, M. Xiao, Y.-H. Ma, Z. Chen, Analytical Chemistry 2017, 89, 1466. 
[37] Z. Chen, Progress in polymer science 2010, 35, 111376.

[38] Z. Hu, F. M. Kerton, Applied Catalysis A: General 2012, 413332.

[39] J. M. Gichumbi, H. B. Friedrich, B. Omondi, Journal of Organometallic Chemistry $2016,80887$.

[40] N. Tsaulwayo, R. T. Kumah, S. O. Ojwach, Polyhedron 2021, 197115034.

[41] J. Pignanelli, B. Billet, M. Straeten, M. Prado, K. Schlingman, M. J. Ahamed, S. Rondeau-Gagné, Soft Matter 2019, 15, 387654.

[42] L.-H. Cai, T. E. Kodger, R. E. Guerra, A. F. Pegoraro, M. Rubinstein, D. A. Weitz, Advanced Materials 2015, 27, 355132.

[43] J. Pignanelli, Z. Qian, X. Gu, M. J. Ahamed, S. Rondeau-Gagné, New Journal of Chemistry 2020, 44, 218977.

[44] D.-P. Wang, J.-C. Lai, H.-Y. Lai, S.-R. Mo, K.-Y. Zeng, C.-H. Li, J.-L. Zuo, Inorganic Chemistry 2018, 57, 63232.

[45] P. H. Budzelaar, B. de Bruin, A. W. Gal, K. Wieghardt, J. H. van Lenthe, Inorganic Chemistry 2001, 40, 184649 .

[46] L. Wang, Q. Ji, T. Glass, T. Ward, J. McGrath, M. Muggli, G. Burns, U. Sorathia, Polymer 2000, 41, 135083.

[47] Z. Zulu, G. S. Nyamato, T. A. Tshabalala, S. O. Ojwach, Inorganica Chimica Acta 2020, 501 119270.

[48] T. Chu, L. Belding, A. van der Est, T. Dudding, I. Korobkov, G. I. Nikonov, Angewandte Chemie International Edition 2014, 53, 102711.

[49] Y. Vidavsky, M. R. Buche, Z. M. Sparrow, X. Zhang, S. J. Yang, R. A. DiStasio Jr, M. N. Silberstein, Macromolecules 2020, 53, 62021.

[50] C.-H. Li, J.-L. Zuo, Advanced Materials 2020, 32, 271903762.

[51] J. Drelich, Surface Innovations 2013, 1, 4248.

[52] J. K. Park, J. Ryu, B. C. Koo, S. Lee, K. H. Kang, Soft Matter 2012, 8, 4711889.

[53] C. Chen, J. Wang, Z. Chen, Langmuir 2004, 20, 2310186.

[54] C. Leng, S. Sun, K. Zhang, S. Jiang, Z. Chen, Acta biomaterialia 2016, 406.

[55] C. Leng, X. Han, Q. Shao, Y. Zhu, Y. Li, S. Jiang, Z. Chen, The Journal of Physical Chemistry C 2014, 118, 2915840.

[56] Y.-L. Rao, V. Feig, X. Gu, G.-J. Nathan Wang, Z. Bao, Journal of Polymer Science Part A: Polymer Chemistry 2017, 55, 183110.

[57] R. Díaz-Torres, S. Alvarez, Dalton Transactions 2011, 40, 4010742.

[58] X. Zhang, Y. Vidavsky, S. Aharonovich, S. J. Yang, M. R. Buche, C. E. Diesendruck, M. N. Silberstein, Soft Matter 2020, 16, 378591.

[59] H.-J. Wang, X.-K. Xi, A. Kleinhammes, Y. Wu, Science 2008, 322, 589880.

[60] M. E. Callow, R. L. Fletcher, International Biodeterioration E Biodegradation 1994, 34, 3-4 333.

[61] M. Inutsuka, H. Tanoue, N. L. Yamada, K. Ito, H. Yokoyama, RSC Advances 2017, 7, 2817202.

[62] M. P. Schultz, J. A. Finlay, M. E. Callow, J. A. Callow, Biofouling 2000, 15, 4243. 
[63] S. Zhang, J. S. Andre, L. Hsu, A. Toolis, S. L. Esarey, B. Li, Z. Chen, Macromolecules 2020, 53, 22 10189.

[64] M. Xiao, S. Joglekar, X. Zhang, J. Jasensky, J. Ma, Q. Cui, L. J. Guo, Z. Chen, Journal of the American Chemical Society 2017, 139, 93378.

[65] H. Huang, C. Zhang, R. Crisci, T. Lu, H.-C. Hung, M. S. J. Sajib, P. Sarker, J. Ma, T. Wei, S. Jiang, et al., Journal of the American Chemical Society 2021. 